\title{
APPLETS EN LA ENSEÑANZA DE LA FÍSICA: UN ANÁLISIS DE LAS CARACTERÍSTICAS TECNOLÓGICAS Y DISCIPLINARES
}

\author{
María José Bouciguez y Graciela Santos \\ Universidad Nacional del Centro de la Provincia de Buenos Aires, Argentina. \\ E-mail:mjbouci@exa.unicen.edu.ar
}

[Recibido en Marzo de 2009, aceptado en Septiembre de 2009]

\begin{abstract}
RESUMEN
Un applet proporciona un andamiaje para conceptualizar fenómenos dinámicos y permite relacionar el modelo científico, las matemáticas y el sentido común. En este artículo describimos características tecnológicas y disciplinares de applets de onda, que han sido seleccionados en Internet. Se ha elaborado un conjunto de categorías para describir las posibilidades interactivas de los applets sobre los dos aspectos mencionados anteriormente. Los resultados que se presentan corresponden a una primera etapa de análisis, y muestran una amplia gama de elementos potencialmente facilitadores de las interacciones del alumno, desde la visualización de gráficos de variables a pequeños micromundos. Las categorías elaboradas pueden ser un instrumento útil para analizar applets, y podrían considerarse como insumos para otras investigaciones educativas, así como también podrían ser una ayuda a los profesores en el proceso de selección de simulaciones.
\end{abstract}

Palabras claves: Applet; interactividad; herramientas cognitivas; modelo científico; movimiento ondulatorio;

\section{APPLETS EN LA ENSEÑANZA}

El paradigma educativo de la nueva sociedad de la información se puede caracterizar por modelos constructivistas de aprendizaje y entornos enriquecidos tecnológicamente (García Barneto y Gil Martín, 2006). La enseñanza de la Física es una de las principales áreas que ha utilizado las potencialidades de la computadora para el desarrollo de nuevas metodologías de enseñanza, motivando un campo de investigación multidisciplinario donde se conjugan la Física, la enseñanza de la Física y la Informática. Esto lo muestra la gran cantidad y variedad de aplicaciones que se han desarrollado.

A la hora de concretizar la enseñanza en una situación de clase, se presenta un doble desafío entre articular lo que el alumno ya sabe y lo que se le presenta como nuevo, de manera que lo nuevo no sea tan complejo que no pueda afrontarlo. Muchos trabajos dan cuenta que la mediación entre el conocimiento científico y la comprensión es una tarea muy compleja (Pozo y Gómez Crespo, 1998). Se requiere apertura, 
flexibilidad y, sobre todo, claridad conceptual para diseñar apropiadas actividades de aprendizaje que promuevan, principalmente, el razonamiento cualitativo.

Una de las dificultades que enfrenta la enseñanza de la Física en el nivel de educación secundaria es que los alumnos aún no están en condiciones de realizar y comprender los necesarios procesos de abstracción que implica la matemática involucrada en muchos fenómenos físicos. Por ejemplo, la enseñanza del movimiento ondulatorio se ve oscurecida por la matemática requerida. Muchas veces, este hecho pesa al momento de tomar decisiones didácticas, eligiendo no abordar el tema, antes de dar un tratamiento conceptual insuficiente y que resulte ininteligible a los alumnos. Para fenómenos complejos, como el movimiento ondulatorio, donde es clave y poco intuitiva la evolución temporal de las variables implicadas, resulta complicado diseñar actividades de aprendizaje conceptualmente productivas con lápiz y papel o en el laboratorio.

La tecnología desarrollada en torno a Internet ofrece ricos, simples y gratuitos recursos educativos. Las aplicaciones informáticas, por ejemplo los applets, pueden servir de soporte para el aprendizaje cualitativo, mediando procesos de modelado y permitiendo el abordaje de diferentes tipos de problemas. Esto puede facilitar la tarea de los profesores cuya intencionalidad didáctica sea que "los estudiantes hagan ciencia de una manera exploratoria y constructiva" (Esquembre, 2005).

Internet posibilita acceder a una gran cantidad de materiales interesantes que, de otro modo, estarían fuera de nuestro alcance. Tal es el caso de la amplia oferta de pequeñas simulaciones (applets) disponibles para la mayoría de los fenómenos físicos que se estudian en clases de física. Un applet es un pequeño programa en lenguaje Java que se puede transmitir por Internet, constituyendo un elemento más de una página Web, como una imagen o un texto. Cuando el navegador carga la página Web, el applet insertado en dicha página se carga y se ejecuta.

Existen estudios que se concentran en analizar el resultado de los aprendizajes cuando se utilizan simulaciones. Tal es el caso de la investigación desarrollada por Garcia Barneto y Bolivar Raya (2005), cuyo objetivo fue valorar la eficacia de las simulaciones informáticas en la producción de aprendizajes significativos. Esta investigación se basa en la hipótesis de que "Los alumnos que hacen uso de simulaciones interactivas (applets Java) en un contexto investigativo mediado por ordenador, unen al aumento del interés una mejora en la significatividad del aprendizaje de los conceptos físicos" (García Barneto y Bolivar Raya, 2005: 2). Para su comprobación, realizaron la experiencia en la asignatura de Física (nivel universitario) durante el tratamiento de los temas movimiento armónico simple y ondulatorio; y se llegó a la conclusión de que las simulaciones interactivas mejoran "realmente el aprendizaje de los conceptos físicos, sin embargo, queda margen de progreso y, mientras no se perfeccionen los aspectos educativos de las simulaciones, corremos el riesgo de provocar errores no deseados" (García Barneto y Bolivar Raya, 2005: 4). Un año más tarde, continuando esta línea de investigación se publica un trabajo donde se reafirma la contribución positiva de las simulaciones interactivas en el proceso de enseñanza y aprendizaje y, se enfatiza que la incorporación de las simulaciones informáticas a la enseñanza de la física debe entenderse como un problema tecnológico y didáctico. Además se proporcionan algunas pautas orientativas para el diseño de entornos de aprendizaje 
basados en simulaciones informáticas. En dicha publicación también se comenta el proyecto de creación de un entorno de aprendizaje constructivista, orientado al tratamiento de los movimientos armónico simple y ondulatorio (García Barneto y Gil Martín, 2006).

En esta comunicación se presenta un estudio que intenta describir y categorizar las características tecnológicas y disciplinares de applets disponibles en Internet, con el objeto de colaborar en la elección de una simulación que pueda servir de apoyo para la enseñanza del movimiento oscilatorio en un sistema abierto. Este trabajo se encuadra en el estudio acerca de las tecnologías educativas con soporte informático para la conceptualización en Física.

\section{MARCO CONCEPTUAL}

En un enfoque social, como sugieren los trabajos más recientes en didáctica de las ciencias y la matemática (Johsua y Dupin, 2005), se considera que el alumno construye sus conocimientos en interacción con su entorno físico y social; que su producción depende, no sólo de la estructura interna de sus conocimientos, sino también del tipo específico de actividad o situación-problema que se le propone y de las posibles interacciones con sus pares. Cuando se involucra a los alumnos en actividades que les son significativas y con contenidos que les son relevantes se generan las condiciones para que se predispongan a aprender (Moreira, 2005). Según Collins (1997) la abstracción y transferencia son posibles cuando el alumno ha experimentado la aplicación de sus conocimientos en una actividad plena de sentido y en contextos variados que facilitan la generalización y la reflexión sobre la práctica.

Aquí queremos destacar que todas las actividades humanas, entre ellas las de aprendizaje, están mediadas por instrumentos, considerando que las herramientas y los símbolos son aspectos de un mismo fenómeno que se conjugan en todo artefacto cultural (Cole y Engeströn, 2001). Desde esta perspectiva sociohistórica, la utilización de la computadora adquiere relevancia pedagógica como herramienta de mediación privilegiada entre las actividades de enseñanza y la asimilación cognitiva. En realidad son las aplicaciones informáticas quienes generan formas personales y sociales de recepción e interacción, ofreciendo posibilidades para hacer distintas tareas (Rodríguez Illera, 2004) y facilitando usos pedagógicos múltiples. Las aplicaciones informáticas poseen una riqueza potencial para robustecer y complementar las capacidades cognitivas que ponen en juego los alumnos al resolver una tarea. Jonassen (2000) las considera herramientas cognitivas por sus funciones para visualizar, organizar, automatizar o suplantar procesos cognitivos específicos de nivel inferior, permitiendo así que los alumnos centren su actividad cognitiva en solucionar el problema entre manos interactuando en el ambiente de aprendizaje.

Las simulaciones computacionales facilitan la representación dinámica del funcionamiento de un sistema y la visualización de procesos, mostrando la evolución del sistema representado y la interacción entre los componentes o consecuencias de tales interacciones dinámica (Pontes, 2005). Esta posibilidad de manipular y transformar objetos en el espacio de la interfaz, comprometido un conjunto diferente de competencias cognitivas (Jonassen y Carr, 2000) en comparación con el uso 
pedagógico de otros recursos, le otorga a las actividades educativas cierto grado de fortalecimiento pedagógico. En otras palabras, los applets resultan un modo de involucrar a los estudiantes en el manejo de una amplia gama de códigos científicos y tecnológicos. Una opción válida para acompañar las propuestas educativas con miras a contribuir en la formación de ciudadanos alfabetizados y críticos (Acevedo Díaz, 2004).

Asumimos que una actividad de aprendizaje con applets tiene por finalidad interactuar con el modelo científico en él representado. Y por tal razón, consideramos importante identificar y caracterizar el abordaje que se hace del modelo científico en un applet y los componentes tecnológicos que se han incluido para favorecer la interacción.

\section{Representación del modelo científico}

Para caracterizar e identificar la representación del modelo científico plasmado en un applet, se consideró el "esquema de modelado" propuesto por Halloun (2000, 2006, 2007), en su Teoría de Modelado en Educación en Ciencias (TMEC). El autor asume que los estudiantes elaboran el conocimiento experiencial acerca de las realidades físicas mediante un ínterjuego entre sus propias ideas sobre el mundo físico y los patrones particulares de ese mundo. También propone un conjunto de instrumentos para ordenar el contenido de los modelos científicos y sus componentes conceptuales, lo que facilita conservar el rigor científico en las transposiciones didácticas. El esquema de modelado es una plantilla de organización que asegura que una concepción y, especialmente, un modelo es elaborado conservando las características primarias, e integrado coherentemente en una teoría dada (Halloun, 2007). Este instrumento se estimó valioso para analizar las características disciplinares de los applets porque ofrece reglas para evaluar, directa o indirectamente, la concepción científica o modelo científico subyacente.

A continuación se comentan brevemente los componentes de un modelo en el marco de esta teoría. Según el autor, el modelado de una situación física requiere definir un sistema físico que incluya aquellas entidades que caractericen las propiedades físicas en estudio (Halloun, 2007). Las entidades y propiedades más salientes de un sistema físico son representadas en el modelo científico por entidades y propiedades conceptuales. Las entidades conceptuales representan objetos o agentes físicos, a menudo mediante un concepto geométrico que representa los aspectos morfológicos primarios. Las propiedades conceptuales, a los que denomina descriptores, representan propiedades primarias comunes de la estructura y comportamiento del sistema. Los descriptores se pueden agrupar en dos categorías: descriptores de objetos y descriptores de interacciones (Halloun, 2006). Un descriptor de objeto representa una propiedad característica de una entidad conceptual, y puede ser intrínseco o de estado. Un descriptor intrínseco, se refiere a un parámetro en el lenguaje matemático y representa una propiedad característica que usualmente no es afectada por el comportamiento del cuerpo (o sistema) o por su interacción con otros cuerpos. En mecánica clásica, son ejemplos de descriptores intrínsecos la masa, carga y las dimensiones. Un descriptor de estado se refiere a una variable en lenguaje matemático y representa una propiedad que caracteriza el comportamiento de un cuerpo, que puede variar durante la interacción con otros cuerpos (velocidad, momentum, corriente eléctrica). Por su parte, un descriptor interacción representa las 
acciones mutuas entre dos o más cuerpos físicos, por ejemplo, en mecánica clásica, fuerza, campo, energía, pudiendo ser estas internas o externas, según sea definido el sistema para representar convenientemente las acciones mutuas de los objetos.

\section{Componentes tecnológicos}

La potencialidad de un applet como herramienta educativa se manifiesta en la calidad de su interfaz gráfica interactiva. En general se entiende por interactividad la actividad y/o acción y/o comunicación entre personas, sea o no mediada por alguna tecnología; pero en el contexto tecnológico este término guarda una estrecha relación con la interacción de las personas con la computadora (Almeida Barreto, Piazzalunga, Guimarães Ribeiro, Casemiro Dalla y Leon Filho, 2003; Teo, Oh, Liu y Wei, 2003; Evans y Gibbons, 2007).

El concepto de interactividad implica un proceso de comunicación entre el usuario y la máquina, incluyendo las acciones del usuario y la respuesta de la máquina. Una buena interactividad se puede lograr cuando la aplicación informática brinda un máximo de posibilidades de comunicación y posee un tiempo de respuesta corto. Lo anterior concuerda con el modelo de interacción formulado por Evans y Sabry (Evans y Gibbons, 2007), para describir los modos de interactividad que promueve una aplicación informática y clarificar el significado de un sistema interactivo. Según este modelo, una interacción implica una secuencia de tres acciones entre dos agentes: iniciación, respuesta y reacción. La iniciación implica la invitación del primer agente al segundo para realizar una entrada; la respuesta se corresponde con la acción del segundo agente para proporcionar la entrada requerida y; la reacción o feedback es la acción realizada por el primer agente, presentando nueva información como devolución.

Cuando un estudiante interactúa con un applet, es a través de la interfaz gráfica de usuario que el segundo induce al primero a iniciar el proceso interactivo y donde, también, se manifiesta la respuesta. En la interfaz se ubican los distintos controles, mediante los que el usuario le comunica a la aplicación computacional sus decisiones. Pero también en la interfaz gráfica se encuentran los componentes tecnológicos por los cuales el applet muestra el comportamiento del modelo computacional en respuesta a la acción del usuario, utilizando diferentes representaciones visuales. Según Minguell (2000):

"La existencia de recursos que permiten que el usuario establezca un proceso de actuación participativa-comunicativa con los materiales será lo que definirá el grado de interactividad del producto. Así, un elevado nivel de intervención-decisión concedido al usuario, la existencia de un amplio abanico de opciones de acceso a la información, una gran sencillez en el modo de comunicarse con el producto, y una gran rapidez en la realización de los procesos (a nivel técnico), aumentaran el nivel de interactividad de la herramienta.

Así, si se incrementa la interactividad de las aplicaciones informáticas, ampliando las posibilidades del usuario para tomar decisiones en relación a "qué hacer o buscar" y "cómo hacerlo", y se consigue aumentar el grado de eficacia de la aplicación al "obedecer" las instrucciones que de el sujeto, se podrá decir que se incrementa el nivel de interactividad." (Minguell, 2000: 26) 


\section{METODOLOGÍA DE BÚSQUEDA, SELECCIÓN Y ANÁLISIS DE APPLETS}

Internet permite un intercambio ilimitado de información, siendo actualmente una importante fuente de recursos. Al buscar información en la Web es necesario evaluar lo que allí encontramos y aplicar ciertos criterios para identificar lo valioso y útil. $\mathrm{A}$ diferencia de los productos que antes de salir al mercado reciben una evaluación crítica para verificar su cumplimiento con ciertas normas de calidad, los contenidos de las páginas y sitios de Internet no están sometidos a ningún tipo de monitoreo antes de formar parte de la World Wide Web. Por esta razón, con el objeto de indagar las características de las aplicaciones Java de la Web, el primer paso consistió en definir criterios para la búsqueda de applets, a partir de una adecuación de los criterios de búsqueda de información en Internet.

A continuación, se presenta el proceso propuesto para la búsqueda, selección y análisis de applets en Internet sobre el tema movimiento ondulatorio.

\section{Criterios de búsqueda y selección de applets}

En primer lugar, se planteó, haciendo uso de los tres buscadores más conocidos: Google, Yahoo y Altavista, la siguiente cadena de palabras claves, en español e inglés: [applets+física+"movimiento ondulatorio"+ondas]. Con el resultado de la búsqueda, se elaboró una lista de sitios Web que presentan applets relacionados al tema movimiento ondulatorio.

Para seleccionar los applet con los que se realizaría el estudio de características disciplinares y tecnológicas, se valoró la fiabilidad del origen (Bohigas, Jaén y Novell, 2003) y cubrimiento de los sitios Web encontrados.

Con fiabilidad del origen se tuvo en cuenta la procedencia, creador y/o aval del sitio que garantice la idoneidad y legitimidad del mismo. A la vez, se evaluó la concordancia de la representación visual y funcional que presenta el applet (elementos visuales para mostrar los resultados: animación, valores de variables, tablas, etc.) con el modelo científicamente concensuado. $Y$ con cubrimiento se consideró si se puede acceder al material sin limitantes como el pago de suscripción, la tecnología del navegador, y/o requisitos del software y/o hardware, y en caso de requerir un software especial, se analiza si se ofrece algún tipo de asesoramiento sobre su adquisición e instalación.

\section{Análisis de las características disciplinares y tecnológicas de los applets}

El presente estudio se encuadra dentro de un análisis de tipo cualitativo, asumiendo como unidad de análisis el applet. Dado que existe una fuerte vinculación funcional entre los componentes disciplinares y tecnológicos, el análisis se aborda bajo dos ejes: disciplinar y tecnológico. Una diferenciación que se asume no excluyente, debido a que la interacción con una entidad o propiedad conceptual es mediada por un componente tecnológico; y por tanto la pertenencia a las categorías del eje disciplinar se decide por la visualización y funcionalidad de dichos componentes.

Las categorías de análisis correspondientes al eje disciplinar se listan y describen en la Tabla 1. Se elaboraron a partir de algunos elementos teóricos tomados, en sentido amplio, del esquema de modelado de Halloun (2006). Se asociaron y estructuraron en 
concordancia con las entidades y las propiedades conceptuales del modelo que se estudia, para intentar describir las características del modelo científico que representa un applet.

\begin{tabular}{|c|c|c|}
\hline Categorías & Códigos & Descripción \\
\hline $\begin{array}{l}\text { Representación de } \\
\text { Entidades } \\
\text { Conceptuales del } \\
\text { modelo científico }\end{array}$ & DEC & $\begin{array}{l}\text { Desde la perspectiva de la TMEC, la entidad conceptual que se } \\
\text { significa en el modelo científico que se espera que representen los } \\
\text { applets es de onda. La pertenencia a esta categoría se considera } \\
\text { cuando se basa en una representación prototípica de onda armónica } \\
\text { simple en un sistema abierto. }\end{array}$ \\
\hline $\begin{array}{l}\text { Representación de } \\
\text { Agentes Externos del } \\
\text { sistema físico }\end{array}$ & DAE & $\begin{array}{l}\text { Una tarea primordial para dar explicación a un fenómeno físico es } \\
\text { delimitar el sistema físico y el ambiente. Sería esperable algún tipo } \\
\text { de representación de los agentes externos al sistema. Por ejemplo, } \\
\text { en el caso aquí analizado, algún componente (imagen o símbolo) } \\
\text { asociado a la fuente que produce la perturbación en el medio. }\end{array}$ \\
\hline $\begin{array}{l}\text { Referencia a Modelos } \\
\text { Básicos relacionados }\end{array}$ & DMB & $\begin{array}{l}\text { Proveer elementos para relacionar el modelo en estudio con los } \\
\text { modelos ya conocidos por el alumno es una estrategia recomendada } \\
\text { en muchos estudios sobre la enseñanza y aprendizaje de las } \\
\text { ciencias. En este caso particular, la partícula es la entidad conceptual } \\
\text { de los dos modelos básicos relacionados y, por tanto, se espera } \\
\text { alguna referencia al movimiento oscilatorio de un punto del medio } \\
\text { perturbado. }\end{array}$ \\
\hline $\begin{array}{l}\text { Presencia de } \\
\text { elementos que } \\
\text { promueven } \\
\text { Relacionar Modelos }\end{array}$ & DRM & $\begin{array}{l}\text { Para promover la relación entre modelos, además de los objetos } \\
\text { sería deseable contar con indicadores de las propiedades de las } \\
\text { correspondientes entidades conceptuales. Por ejemplo, indicadores } \\
\text { de la posición de equilibrio, dirección de movimiento para relacionar } \\
\text { el movimiento ondulatorio y el movimiento de una partícula del medio. }\end{array}$ \\
\hline $\begin{array}{l}\text { Manifestación de } \\
\text { Propiedades } \\
\text { Intrínsecas }\end{array}$ & DPI & $\begin{array}{l}\text { Otro aspecto que requiere atención para comprender un modelo } \\
\text { cientifico es identificar las propiedades que no son afectadas por el } \\
\text { comportamiento del cuerpo (o sistema) o por su interacción con otros } \\
\text { cuerpos. En este caso, se espera alguna manifestación de las } \\
\text { propiedades del medio (elástica e inercial). }\end{array}$ \\
\hline $\begin{array}{l}\text { Tratamiento de } \\
\text { Propiedades de } \\
\text { Estado }\end{array}$ & DPE & $\begin{array}{l}\text { Las distintas formas de expresar las relaciones entre las variables } \\
\text { facilitan comprender la evolución del sistema físico. En este análisis } \\
\text { se considera si se presentan elementos para el tratamiento y/o } \\
\text { manipulación de la longitud de onda, frecuencia y amplitud. }\end{array}$ \\
\hline $\begin{array}{l}\text { Consideración de los } \\
\text { Descriptores de } \\
\text { Interacción }\end{array}$ & DDI & $\begin{array}{l}\text { Es posible entender un modelo y su evolución cuando se analizan las } \\
\text { interacciones entre los objetos del sistema físico, o de estos con los } \\
\text { agentes externos. Por ejemplo, en el movimiento ondulatorio las } \\
\text { interacciones entre las partículas del medio y de estas con la fuente } \\
\text { de perturbación, que dan lugar al transporte de energía e impulso, } \\
\text { pero no de materia. }\end{array}$ \\
\hline
\end{tabular}

Tabla 1.- Categorías, códigos, descripción e indicadores correspondientes al eje Disciplinar.

En particular, aquí se consideraron applets que simulan el movimiento ondulatorio en un sistema físico abierto, generado por una fuente que oscila con un movimiento armónico simple. Dado que el movimiento ondulatorio implica interacciones entre un gran número de partículas, el modelo se entiende como modelo emergente de los modelos oscilador armónico simple y partícula bajo una interacción impulsiva, que son 
modelos básicos de la teoría Newtoniana de la mecánica clásica (Halloun, 2007). Además, que las ondas tienen la importante propiedad de transportar energía e impulso a través del medio, sin transporte de materia.

Como se mencionó antes, el escenario de la interacción con un applet es la interfaz gráfica de usuario, conteniendo componentes asociados con la visualización (acción ver) y el control (acción hacer). Teniendo en cuenta estos dos tipos de componentes se elaboraron las categorías de análisis correspondientes al eje tecnológico, las que se listan y describen en la Tabla 2.

\begin{tabular}{|c|c|c|}
\hline Categorías & Códigos & Descripción \\
\hline $\begin{array}{l}\text { Representación } \\
\text { Análoga }\end{array}$ & TRA & $\begin{array}{l}\text { Visualización dinámica del modelo científico. Conjunto de elementos } \\
\text { iconográficos que poseen atributos morfológicos análogos a los que } \\
\text { intervienen en el fenómeno científico y cuyo comportamiento, en } \\
\text { conjunto, representa visualmente el del modelo científico } \\
\text { subyacente. }\end{array}$ \\
\hline $\begin{array}{l}\text { Representación } \\
\text { Gráfica }\end{array}$ & TRG & $\begin{array}{l}\text { Visualización dinámica del modelo científico. Representación gráfica } \\
\text { matemática de relaciones entre diferentes variables del modelo. }\end{array}$ \\
\hline $\begin{array}{l}\text { Manipulación de } \\
\text { Parámetros }\end{array}$ & TMP & $\begin{array}{l}\text { Objetos iconográficos que permiten modificar el valor de un } \\
\text { parámetro y/o condición inicial del modelo y definir las condiciones } \\
\text { de funcionamiento del mismo. }\end{array}$ \\
\hline Control Play & TCP & $\begin{array}{l}\text { Indicadores que manifiestan la posibilidad de que el usuario pueda } \\
\text { decidir cuando comenzar la ejecución de la simulación desde los } \\
\text { valores iniciales predefinidos. }\end{array}$ \\
\hline Control Stop/Pausa & TCS & $\begin{array}{l}\text { Indicadores que manifiestan la posibilidad del usuario de detener la } \\
\text { simulación en un instante de tiempo deseado, y de reanudar la } \\
\text { misma cuando haya sido detenida previamente. }\end{array}$ \\
\hline Avance por Paso & TAP & $\begin{array}{l}\text { Indicadores que manifiestan la posibilidad de que el usuario pueda } \\
\text { decidir avanzar un paso de tiempo }(t+d t) \text { en la evolución temporal del } \\
\text { proceso de simulación. }\end{array}$ \\
\hline Retroceso por Paso & TRP & $\begin{array}{l}\text { Indicadores que manifiestan la posibilidad de que el usuario pueda } \\
\text { decidir retroceder un paso de tiempo (t-dt) y visualizar el estado } \\
\text { anterior del sistema simulado. }\end{array}$ \\
\hline $\begin{array}{l}\text { Control de Inicio o } \\
\text { reset }\end{array}$ & TCI & $\begin{array}{l}\text { Indicadores que manifiestan la posibilidad de que el usuario pueda } \\
\text { decidir recomenzar la ejecución de la simulación y volver todas las } \\
\text { variables a sus valores iniciales. }\end{array}$ \\
\hline
\end{tabular}

Tabla 2.- Categorías, códigos e indicadores correspondientes al eje Tecnológico.

Las animaciones son representaciones dinámicas, que a diferencia de las representaciones estáticas como las que se encuentran en los libros, favorecen la interpretación de fenómenos dinámicos. El concepto de onda, se puede objetivar visualmente mediante una representación gráfica o análoga animada que recree visual y funcionalmente una situación real conocida. Los movimientos y cambios en la animación son conducidos por las variables utilizadas en el modelo matemático que define el comportamiento del sistema simulado.

En la interfaz también se encuentran aquellos elementos que permiten modificar el valor de los parámetros y/o condiciones iniciales de un determinado fenómeno físico. 
Un tercer grupo de componentes de la interfaz son los elementos que permiten controlar el proceso de la simulación.

\section{RESULTADOS Y DISCUSIÓN DEL PROCESO DE BÚSQUEDA Y ANÁLISIS}

La búsqueda de sitios con applets sobre el tema movimiento ondulatorio permitió obtener un conjunto de 12 sitios Web (Tabla 3). Todos los sitios, de acuerdo a la clasificación propuesta por Área Moreira (2003), son del tipo material didáctico Web. Entre los principales atributos se puede destacar:

- En todos se infiere una intencionalidad de promover en los usuarios el desarrollo de algún proceso de aprendizaje mediante la interacción con un applet.

- La mayoría combina información con una propuesta de actividad interactiva con el applet.

- Ocho sitios conforman una colección de applets (CA) sin que necesariamente formen parte de una propuesta de enseñanza para una temática en particular.

- El abordaje de la temática varía de uno a otro, dependiendo del nivel educativo para el que se han diseñado.

- Muchos contienen información conectada hipertextualmente, integrando distintas formas de representación simbólica, como por ejemplo textual, gráfica, audiovisual, icónica, etc. (Área Moreira, 2003).

- Cinco pueden considerarse unidades didácticas interactivas (UDIs), porque "integran las simulaciones en un contexto educativo que da continuidad y coherencia al conjunto de la obra" (García Barneto y Gil Martín, 2006).

Dado que en este trabajo nos proponemos analizar applets que aborden el movimiento ondulatorio en sistemas abiertos se escogieron solamente 10 de esos sitios y de ellos se seleccionaron solo aquellas páginas que presentaban applets referidos al tema.

El análisis de los 15 applets seleccionados según las categorías del eje disciplinar y el eje tecnológico se presentan en la Tabla 4. Los resultados (cruz) indican la pertenencia del applet a una categoría dada de acuerdo a la descripción e indicadores comentados en las Tablas 1 y 2.

Debido a la estrecha vinculación entre las categorías de ambos ejes, y especialmente la dependencia de las categorías disciplinares con los componentes que se muestran en la interfaz gráfica interactiva, se describen y discuten los resultados obtenidos para ambos ejes de manera conjunta.

De un total de 15 applets analizados, en la mayoría (13) se trabaja con una representación análoga del fenómeno simulado, dirigiendo la atención del usuario hacia la onda como entidad conceptual objeto de estudio. En dos (2) se utiliza como única representación animada un gráfico matemático, poniendo énfasis en una variable de estado. Respecto a las entidades conceptuales externas al sistema (DAE), que involucra el modelo científico que se espera que los applets representen, se encontró poca referencia a la fuente de perturbación (agente externo), solo visualizada en dos (2) casos. Esta característica podría tener justificación dado que se 
está representando el modelo de onda ideal (única frecuencia, longitud de onda, rapidez de onda y amplitud, e infinitamente larga), mostrando solo una parte del medio.

\begin{tabular}{|c|c|c|c|}
\hline Sitio & Tipo & Applet & URL \\
\hline $\begin{array}{l}\text { NTNUJAVA Virtual } \\
\text { Physics Laboratory (Java } \\
\text { Simulations in Physics) } \\
\text { Autor: Hwang, F. }\end{array}$ & CA & $\begin{array}{l}\text { (1)Transverse Wave and } \\
\text { Longitudinal Wave }\end{array}$ & $\begin{array}{l}\text { http://www.phy.ntnu.edu.tw/ntnujava/index. } \\
\text { php?topic=14.msg80 }\end{array}$ \\
\hline \multirow{2}{*}{$\begin{array}{l}\text { Física con ordenador: } \\
\text { Curso interactivo de física } \\
\text { en Internet } \\
\text { Autor: Franco García, Á. }\end{array}$} & \multirow[b]{2}{*}{ UDI } & $\begin{array}{l}\text { (2) Descripción de la } \\
\text { propagación de un M. O. }\end{array}$ & $\begin{array}{l}\text { http://www.sc.ehu.es/sbweb/fisica/ondas/d } \\
\text { escripcion/descripcion.html }\end{array}$ \\
\hline & & (3) Propagación de un MOA & $\begin{array}{l}\text { http://www.sc.ehu.es/sbweb/fisica/ondas/o } \\
\text { ndaArmonica/ondasArmonicas.htm|\#Onda } \\
\text { s\%20longitudinales\%20en\%20una } \\
\text { \%20barra\%20elástica }\end{array}$ \\
\hline \multirow{2}{*}{$\begin{array}{l}\text { Using Java applets to } \\
\text { simulate physics } \\
\text { phenomena. } \\
\text { Autor: Chiu-king Ng. }\end{array}$} & \multirow{2}{*}{ CA } & (4) Transverse Travelling Wave & $\begin{array}{l}\text { http://www.ngsir.netfirms.com/englishhtm/T } \\
\text { waveA.htm }\end{array}$ \\
\hline & & $\begin{array}{l}\text { (5) Longitudinal Travelling } \\
\text { Wave }\end{array}$ & $\begin{array}{l}\text { http://www.ngsir.netfirms.com/englishhtm/L } \\
\text { wave.htm }\end{array}$ \\
\hline $\begin{array}{l}\text { Physics Educations } \\
\text { Technology (PhET). } \\
\text { Autores: Dubson, } \\
\text { Adams y Harllow }\end{array}$ & CA & (6) Wave on a string & $\begin{array}{l}\text { http://phet.colorado.edu/simulations/sims.p } \\
\text { hp?sim=Wave on a String }\end{array}$ \\
\hline $\begin{array}{l}\text { Ondas } \\
\text { Autor: García, L. I. }\end{array}$ & UDI & (7) Ondas & $\begin{array}{l}\text { http://web.educastur.princast.es/proyectos/ } \\
\text { fisquiweb/MovOnd/index.htm }\end{array}$ \\
\hline $\begin{array}{l}\text { Laboratorio Virtual } \\
\text { Prisma CNICE }\end{array}$ & $\begin{array}{l}\text { CA / } \\
\text { UDI }\end{array}$ & (8) Ondas & $\begin{array}{l}\text { http://enebro.pntic.mec.es/ fmag0006/op } \\
\text { applet 8.htm }\end{array}$ \\
\hline \multirow{2}{*}{$\begin{array}{l}\text { General Physics Java } \\
\text { Applets. } \\
\text { Autor: Surendranath } \\
\text { Reddy, B. }\end{array}$} & \multirow{2}{*}{$\mathrm{CA}$} & (9) Transverse waves & $\begin{array}{l}\text { http://www.surendranath.org/Applets/Wave } \\
\text { s/Twave01/Twave01Applet.html }\end{array}$ \\
\hline & & (10) Longitudinal waves & $\begin{array}{l}\text { http://www.surendranath.org/Applets/Wave } \\
\text { s/Lwave01/Lwave01Applet.html }\end{array}$ \\
\hline $\begin{array}{l}\text { Physics } \\
\text { Autor: Duke, P. }\end{array}$ & $\mathrm{CA}$ & $\begin{array}{l}\text { (11) Transverse and } \\
\text { Longitudinal Waves }\end{array}$ & $\begin{array}{l}\text { http://www.mrfizzix.com/utilitypage/dukes/ } \\
\underline{\text { wavetrans/WaveTrans.htm }}\end{array}$ \\
\hline $\begin{array}{l}\text { Laboratorio Virtual de } \\
\text { Física } \\
\text { Autor: Casellas, T. }\end{array}$ & $\mathrm{CA}$ & (12) FisLab.net & $\begin{array}{l}\text { http://www.xtec.net/ ocasella/applets/ones/ } \\
\text { appletsol2.htm }\end{array}$ \\
\hline \multirow{3}{*}{$\begin{array}{l}\text { Physics at Indiana } \\
\text { University Southeast } \\
\text { Autor: Forinash, K. }\end{array}$} & \multirow{3}{*}{ UDls } & (13) Sine Waves. & \multirow{3}{*}{ http://physics.ius.edu/ kyle/W/Waves.html } \\
\hline & & (14) Transverse Waves. & \\
\hline & & (15) Longitudinal Waves. & \\
\hline
\end{tabular}

Tabla 3: Sitios seleccionados según fiabilidad de origen y cubrimiento conteniendo Applets sobre el tema movimiento ondulatorio en sistemas abiertos. Además, se indica si son un conjunto de applets (CA) o Unidades Didácticas Interactivas (UDIs). 


\begin{tabular}{|c|c|c|c|c|c|c|c|c|c|c|c|c|c|c|c|}
\hline \multirow{2}{*}{ Applet } & \multicolumn{7}{|c|}{ Eje Disciplinar } & \multicolumn{8}{|c|}{ Eje Tecnológico } \\
\hline & DEC & DAE & DMB & DRM & DPI & \begin{tabular}{|l|l|} 
DPE \\
\end{tabular} & DDI & TRA & TRG & TMP & TCP & TCS & TCI & TAP & TRP \\
\hline (1) Hwang, F. & $\mathrm{x}$ & & $x$ & $\mathrm{x}$ & & $\mathrm{x}$ & $\mathrm{x}$ & $x$ & & $\mathrm{x}$ & & $\mathrm{x}$ & & & \\
\hline $\begin{array}{l}\text { (2) Franco } \\
\text { García, A. }\end{array}$ & $x$ & & $x$ & & $\mathrm{x}$ & $x$ & & $x$ & & $x$ & $\mathrm{x}$ & $x$ & $x$ & $x$ & \\
\hline $\begin{array}{l}\text { (3) Franco } \\
\text { García, A. }\end{array}$ & $x$ & & $x$ & & $\mathrm{x}$ & $x$ & & $x$ & $\mathrm{x}$ & $x$ & $\mathrm{x}$ & $x$ & $x$ & $x$ & \\
\hline (4) Chiu-king Ng & $x$ & & $x$ & $x$ & & $x$ & & $x$ & & $x$ & $x$ & $x$ & & $x$ & \\
\hline (5) Chiu-king Ng & $x$ & & $x$ & $x$ & & $x$ & & $x$ & $x$ & $x$ & $x$ & $x$ & & $x$ & \\
\hline $\begin{array}{l}\text { (6) Dubson, } \\
\text { Adams y Harllow }\end{array}$ & $x$ & $\mathrm{x}$ & $\mathrm{x}$ & & $x$ & $x$ & & $x$ & & $x$ & $x$ & $x$ & $x$ & $x$ & \\
\hline (7) García, L. I. & $x$ & $x$ & $x$ & & & $x$ & $x$ & $x$ & & & $x$ & $x$ & & & \\
\hline $\begin{array}{l}\text { (8) Lab Virtual } \\
\text { Prisma CNICE }\end{array}$ & & & & & $x$ & $\mathrm{x}$ & & & $x$ & $x$ & & $x$ & & & \\
\hline $\begin{array}{l}\text { (9) Surendranath } \\
\text { Reddy, B. }\end{array}$ & $x$ & & $\mathrm{x}$ & $x$ & & $\mathrm{x}$ & & $\mathrm{x}$ & & $\mathrm{x}$ & $x$ & $x$ & $\mathrm{x}$ & $x$ & $x$ \\
\hline $\begin{array}{l}\text { (10) } \\
\text { Surendranath } \\
\text { Reddy, B. }\end{array}$ & $x$ & & $\mathrm{x}$ & $x$ & & $\mathrm{x}$ & & $x$ & & $\mathrm{x}$ & $x$ & $x$ & $x$ & $x$ & $x$ \\
\hline (11) Duke, P. & $x$ & & $x$ & $x$ & & & & $\mathrm{x}$ & & & $\mathrm{x}$ & $x$ & $\mathrm{x}$ & & \\
\hline (12) Casellas, T. & $x$ & & $x$ & $\mathrm{x}$ & & $x$ & & $x$ & $x$ & $x$ & $x$ & $x$ & $x$ & $x$ & $x$ \\
\hline (13) Forinash. K. & & & & & & $x$ & & & $x$ & $x$ & $x$ & $x$ & $x$ & $x$ & $x$ \\
\hline (14) Forinash. K. & $x$ & & $x$ & $x$ & & $\mathrm{x}$ & & $x$ & $x$ & & $x$ & $x$ & $x$ & $x$ & $x$ \\
\hline (15) Forinash. K. & $x$ & & $x$ & $x$ & & $\mathrm{x}$ & & $x$ & $x$ & & $x$ & $x$ & $x$ & $x$ & $\mathrm{x}$ \\
\hline $\begin{array}{l}\text { Total por } \\
\text { categoría }\end{array}$ & 13 & 2 & 13 & 9 & 4 & 14 & 2 & 13 & 7 & 11 & 13 & 15 & 10 & 11 & 6 \\
\hline
\end{tabular}

Tabla 4.- Análisis de las características disciplinares y tecnológicas de los applets seleccionados.

La entidad conceptual partícula, componente de los modelos básico (DMB), fue incluida en trece (13) applets. Esto muestra una tendencia (o preocupación) común de los autores por hacer evidente que las partículas que componen el medio donde se propaga la onda tienen un movimiento oscilatorio armónico. Nueve (9) de estos applets también pertenecen a la categoría DRM porque se les ha incluido elementos visuales para representar alguna variable de estado de la partícula seleccionada, por ejemplo la dirección de movimiento o velocidad, asociados principalmente con la intención de facilitar la diferenciación entre ondas longitudinales y transversales. 
interacción, tienen escasa representación. Se podría inferir que a estos aspectos disciplinares se les atribuiría escasa relevancia en la enseñanza del movimiento ondulatorio.

Es posible observar que en todas estas aplicaciones informáticas se considera la posibilidad de poder detener la simulación en un instante de tiempo y continuar desde ese punto cuando el usuario lo desee (TCS). Consideramos que esta característica podría contribuir a elaborar la idea de una onda infinitamente larga y que lo visualizado es una porción del universo afectado por una perturbación.

En 13 de los applets se brinda al alumno la posibilidad de decidir cuando comenzar (TCP) la simulación. Se puede observar en la tabla, que cuando esta opción no esta presente tampoco existe un elemento interactivo que permite reiniciar (TCI) la simulación desde valores iniciales predefinidos. Los applets que no permiten al alumno decidir sobre cuando comenzar la simulación, se ejecutan en cuanto se carga en el navegador la página Web que los contiene, o cuando se modifica un parámetro o selección de visualización. En estos casos, para volver a comenzar la simulación será necesario volver a cargar la página o modificar un parámetro o una opción de visualización.

En 11 de los casos analizados se puede observar de manera más detallada el fenómeno, ya que se le permite al usuario avanzar paso a paso en el tiempo. En cada paso del tiempo se visualiza el nuevo estado del sistema, definido por el valor de un conjunto de variables que cambian con el tiempo. Sin embargo son muy pocos (6) los applets que permiten retroceder en el tiempo y visualizar el estado anterior en el que se encontraba el sistema simulado.

En la Tabla 4, también se puede observar que para el applet número 12 del sitio Laboratorio Virtual de Física (Casellas, T.) se han identificado todas las categorías definidas para el eje tecnológico. Se puede decir que, más allá del abordaje que haga del tema en cuestión, dicho applet provee un elevado nivel de interactividad que le permite al usuario un máximo de posibilidades para explorar y probar hipótesis del fenómeno simulado. Sin embargo, el dibujo del bote sobre la representación gráfica de la onda puede inducir o reforzar la idea que las ondas transportan materia.

Las interfaces de los applets que pertenecen a sitios clasificados como unidades didácticas interactivas (UDIs) presentan los mismos elementos interactivos y utilizan los mismos códigos simbólicos. Esto muestra que han sido diseñados e implementados para ser utilizados de una determinada manera y hacer una interpretación particular del fenómeno. Si el applet es de tipo mostrativo, comprometiendo a que el usuario sólo sea espectador o su intervención se reduzca lo mínimo posible, basta con que en su interfaz exista algún elemento de control que permita poner en marcha la simulación.

Al igual que sucede al leer un tema de libros escritos por distintos autores, se puede inferir en los applets analizados, que debajo de cada diseño e implementación existen diferentes maneras de expresar el mismo fenómeno.

Durante el proceso de análisis se encontró que en algunos casos al interactuar en un cierto orden con dos o más elementos interactivos de la interfaz, se lograba una funcionalidad asociada a una de las categorías definidas. Por ejemplo, en el applet 
numero 9 del sitio General Physics Java Applets (Surendranath Reddy, B.), al realizar la secuencia de acciones: clic en el botón "stop" seguida de un posicionamiento en el punto inicial de la barra de control de avance en el tiempo, y a continuación clic en el botón "start"; se obtenía el comportamiento que se describe para un componente de control reset. En otros casos al interactuar con un elemento interactivo con un código (simbólico o textual) que podría asociarse a una categoría del eje tecnológico, se lograba una acción sobre la simulación equivalente a la lograda con otro tipo de control. Por ejemplo, en el applet numero 2 del sitio Física con ordenador: Curso interactivo de física en Internet (Franco García, Á), al hacer clic en el botón "empezar", una vez que la simulación está en curso, se obtenía el comportamiento que se describe para un componente de control reset.

A manera de síntesis, las principales características de los applets analizados serían: presentan una animación de la propagación de una perturbación en un medio, ofrecen variados componentes tecnológicos para manipular las variables longitud de onda, frecuencia y amplitud. En algunos se distingue la intención de salvar un obstáculo de aprendizaje frecuente derivado de la semejanza morfológica entre una onda transversal y la evolución temporal de la posición de un punto del medio, por ejemplo el applet 14 (Transverse Waves de Forinash).

La fuente de perturbación, las propiedades intrínsecas del medio y las interacciones entre las partículas del medio prácticamente no se abordan, mostrando un marcado interés por el modelo matemático que relaciona las variables más que por el fenómeno físico. En cierta forma, este enfoque contribuiría a generar actividades interactivamente más limitadas priorizando los modelos matemáticos al modelo científico (Monroy, Testa y Lombarda, 2006).

Un caso donde ambos modelos se complementan es el applet 6 (Una onda en una cuerda, sitio Web PhET), logrando un alto nivel de interacción conceptual con el fenómeno simulado, permitiendo además redefinir el sistema físico.

\section{CONOCIMIENTO BASE PARA ENSEÑAR CIENCIAS CON TECNOLOGÍA}

Mucho se ha mencionado que las TIC "per se" no producen mejores aprendizajes sino que este depende de una enseñanza que enlaza y fusiona las tareas de aprendizaje, metodología e interacciones; es decir, más que un tema de qué tecnología, es un tema de cómo se utiliza la tecnología (Jiménez, 2009). Una problemática vinculada al conocimiento de los profesores acerca de las tecnologías y los modos de usarlas para promover los aprendizajes.

La práctica de enseñanza consiste en cómo enseñar unos contenidos a unos alumnos en un contexto escolar determinado. Una "combinación entre el conocimiento de la materia a enseñar y el conocimiento pedagógico y didáctico relativo a cómo enseñarla" (Acevedo, 2009), denominado conocimiento didáctico del contenido (Bolívar, 2005; Acevedo, 2009). Cuando se integran las TIC a la práctica de enseñanza se sumaría a el conocimiento tecnológico. Un conocimiento generado por el uso de tecnología para realizar "actividades socialmente situadas" (Lave, 2001). Desde un enfoque sociocultural, estaría vinculado al "hacer" con instrumentos en una relación que, por un lado, transforma el medio y a los mismos instrumentos $y$, por otro, modifica la 
cognición del sujeto (Lion, 2006). Por ejemplo, el conocimiento de alguien que utiliza la tecnología solo para comunicarse vía mail y buscar información en la Web no será el mismo de aquel que participa de la Web social, construye ideas o resuelve problemas en diálogo con la tecnología. Cuanto mayor variedad de actividades se realicen con tecnología mayor será la versatilidad de como se utilicen o recreen. En particular, las simulaciones pueden ser consideradas en la enseñanza de las ciencias como instrumentos de pensamiento calificados con potencialidad para representar modelos y visualizar los procesos dinámicos y los efectos de modificar variables y parámetros del modelo simulado (Pontes, 2005).

En base a estos conceptos, consideramos que el conocimiento tecnológico es un factor importante de influencia en la práctica de la enseñanza, vinculando los contenidos, las estrategias de enseñanza y los procesos de aprendizaje. Específicamente, y a modo de ejemplo en relación al análisis que aquí se presenta, no se trata solo de buscar un applet en la Web relacionado con el contenido a enseñar sino que se trata de seleccionar un applet (o conjunto de applets) de acuerdo al conocimiento didáctico del contenido, considerando además de los aspectos aquí estudiados, disciplinares y tecnológicos, los aspectos pedagógicos, como por ejemplo cuáles son las concepciones y modos de razonamientos de los estudiantes (Johsua y Dupin, 2005).

En síntesis, el análisis cualitativo de las características disciplinares y tecnológicas que aquí se presenta podría servir como un instrumento de análisis para que los profesores puedan valorar componentes disciplinares y tecnológicos de los applets, en el sentido de colaborar en el planeamiento de la práctica de enseñanza. Por ejemplo, si la intencionalidad de la práctica es mostrativa o demostrativa de un fenómeno físico o un aspecto de este, seguramente entre los principales criterios de selección que se plantee el profesor no estará el nivel de interactividad. Si desea hacer un abordaje cuantitativo, poniendo énfasis en las propiedades de estado, variables de estado, tomará en cuenta la presencia de las categorías Tratamiento de Propiedades de Estado, Representación Gráfica y Manipulación de parámetros. Por el contrario, si está interesado en un abordaje cualitativo del fenómeno, con la intencionalidad didáctica que el estudiante pueda explicitar sus concepciones iniciales $y$, a partir de interactuar con el applet, sus pares y profesor, adaptar sus modelos de forma iterativa (Giudice y Galagovsky, 2008), hacia la comprensión del modelo científico que se estudia, tomará en consideración categorías como: Representación de Entidades Conceptuales del modelo científico, Representación de Agentes Externos del sistema físico, Referencia a Modelos Básicos relacionados, Presencia de elementos que promueven Relacionar Modelos, Manifestación de Propiedades Intrínsecas, así como los componentes tecnológicos asociados con la Representación Análoga y los controles de la simulación.

Por lo antes mencionado, el conocimiento base de la enseñanza con tecnología consistiría de una sinergia entre el conocimiento del contenido, el conocimiento pedagógico y didáctico y el conocimiento tecnológico. $Y$, a la vez, también se enseña conocimiento tecnológico para, y en, la acción. 


\section{COMENTARIOS FINALES}

En este trabajo se presenta una propuesta de análisis cualitativo de simulaciones que fue aplicada a applets disponibles en línea referidos al movimiento ondulatorio en sistemas abiertos. La propuesta tiene en cuenta sus características tecnológicas y disciplinares que intenta colaborar con el planeamiento de prácticas de enseñanza de ciencia con simulaciones.

A partir de la búsqueda y selección de applets, se estudian los modos en que, en estos materiales, se aborda el tema (características disciplinares) y los tipos de componentes tecnológicos incluidos en la interfaz gráfica de usuario, para viabilizar la manipulación del modelo científico presentado (características tecnológicas). Ambos aspectos se encuentran vinculados funcionalmente, ya que la interacción con una entidad o propiedad conceptual es mediada por un componente tecnológico.

Las características disciplinares se analizan tomando como referencia el esquema de modelado propuesto por Halloun (2006), que consiste de un instrumento que facilita la identificación y organización de los componentes de un modelo científico. Mientras que las características tecnológicas de los applets son indagadas sobre la base del modelo de interactividad de Evans y Sabry (Evans y Gibbons, 2007), basado en las acciones de iniciación, respuesta y reacción. Se elaboró un instrumento que permite analizar la interactividad, expresada mediante múltiples códigos de visualización en la interfaz gráfica de usuario, que invita al estudiante a interactuar con el modelo científico que el applet representa.

La caracterización obtenida de los applets y la metodología de evaluación bosquejada podrían ser de utilidad para otras investigaciones educativas, así como también colaborar en la toma de decisiones que forman parte de la tarea docente de planeamiento de actividades áulicas.

Una de las cualidades de los applets es la posibilidad que ofrecen de superación de las dificultades que presentan las representaciones estáticas de fenómenos dinámicos (Pintó, Couso y Oro, 2006), cualificándolos como potenciales instrumentos mediadores de conceptualizaciones de Física. En orden a estudiar, desde la perspectiva del estudiante, las características mediacionales de este tipo de simulaciones se prevé indagar sobre los modos de interacción que emplean los estudiantes del nivel de educación media cuando son comprometidos en actividades de aprendizaje que implican interactuar con applets.

\section{Agradecimientos}

Este trabajo ha sido financiado por la Agencia Nacional de Promoción Científica y Tecnológica a través del FONCyT, Contrato de Préstamo BID 1728/OC-AR, Proyecto PICT-05 No: 34479.

\section{REFERENCIAS BIBLIOGRÁFICAS}

Acevedo Díaz, J. (2004). Reflexiones sobre las finalidades de la enseñanza de las ciencias: educación científica para la ciudadanía. Revista Eureka sobre Enseñanza y Divulgación de las Ciencias, 1(1), pp. 3-16. Recuperado el 02 de junio de 2009 de http://www.apac-eureka.org/revista/ 
Almeida Barretto, S. F., Piazzalunga, R., Guimarães Ribeiro, V., Casemiro Dalla, M. B. y Leon Filho, R.M. (2003). Combining interactivity and improved layout while creating educational software for the Web, Computers \& Education, 40 (3), 271-284.

Área Moreira, M. (2003). De los Webs educativos al material didáctico Web. Revista Comunicación y Pedagogía, 188, 32-38.

Bohigas, X. Jaén, X. y Novell, M. (2003). Innovaciones didácticas. Applets en la enseñanza de la física. Revista Electrónica de Enseñanza de las Ciencias, 21 (3), 463-472.

Bolívar, A. (2005). Conocimiento didáctico del contenido y didácticas específicas. Profesorado. Revista de currículum y formación del profesorado, 9 (2), pp. 1-39. Recuperado el 02 de junio de 2009 de http://www.ugr.es/ recfpro/rev92ed.pdf

Cole, M. y Engeströn, Y. (2001). Enfoque histórico-cultural de la cognición distribuida. En Salomón (Ed.). Cogniciones distribuidas. Buenos Aires: Amorrortu editores.

Collins, A. (1997). El potencial de las tecnologías de la Información para la educación. En Vizcarro y León (Eds.). Nuevas tecnologías para el aprendizaje. Madrid: Pirámide.

Esquembre, F. (2005). Creaciones de Simulaciones Interactivas en Java. Aplicaciones a La Enseñanza de La Física. Madrid: Pearson Education, S.A.

Evans, C. y Gibbons, N. (2007). The interactivity effect in multimedia learning. Computers \& Education, 49, 1147-1160.

García Barneto, A. y Bolivar Raya, J. P. (2005). Uso de simulaciones informáticas en la enseñanza de la física: movimientos armónicos simples y ondulatorios. Revista Electrónica de Enseñanza de las Ciencias. Número extra. VII Congreso.

García Barneto, A. y Gil Martín, M. R. (2006). Entornos constructivistas de aprendizaje basados en simulaciones informáticas. Revista Electrónica de Enseñanza de las Ciencias, 5 (2), 304-322.

Giudice, J. y Galagovsky, L. (2008) Modelar la naturaleza discontinua de la materia: una propuesta para la Escuela Media. Revista Electrónica de Enseñanza de las Ciencias Vol. 7 N03. Recuperado 10 de junio de 2009 de http://www.saum.uvigo.es/reec/Volumenes.htm

Halloun, I. (2000). Model-laden inquiry for effective physics instruction. THEMES 1 (4), 339-355.

Halloun, I. (2006). Modeling Theory in Science Education. Netherlands-Holland: Springer.

Halloun, I. (2007). Mediated modeling in science education. Science \& Education, 16 (6-7), 653-697.

Jiménez, J. J. (2009). Biografías de científicas. Una aproximación al papel de la mujer en ciencias desde un enfoque socioconstructivista con el uso de las TIC. Revista 
Eureka sobre Enseñanza y Divulgación de las Ciencias, 6(2), pp. 264-277. Recuperado el 16 de junio de 2009 de http://www.apac-eureka.org/revista/

Johsua, S. y Dupin, J. (2005). Introducción a la Didáctica de las Ciencias y la Matemática. Buenos Aires: Colihue.

Jonassen, D. (2000). El diseño de entornos constructivistas de Aprendizaje. En C. Reigeluth (Ed.). Diseño de la Instrucción. Teorías y modelos. Madrid: Santillana.

Jonassen, D. y Carr, C. (2000). Mindtools: Affording Multiple Knowledge Representations for Learning. En S. P. Lajoie (Ed.). Computer as cognitive tools. Mahwah, NJ: Lawrence Erlbaum Assosiates.

Jonassen, D., Carr, C. y Yueh, H. (1998). Computers as Mindtools for engaging Learners in Critical Thinking, TechTrends, 43(2), 24-32.

Minguell, M. E. (2002). Interactividad e Interacción. Revista Latinoamericana de Tecnología Educativa (RELATEC), 1(1), 23-32.

Monroy, G., Testa, I. y Lombarda, S. (2006). Teaching Wave Physics Through Modeling Images: Use Of Cabri ${ }^{\circ}$ To Address Water Waves Geometrical Models And Basic Laws. En van den Berg, E, Ellermeijer, T. y Slooten, O. (Eds.), Modeling in Physics and Physics Education, Proceedings GIREP Conference 2006, 283-291.

Moreira, M. A. (2005). Aprendizaje Significativo Crítico. Porto Alegre, Brasil: Impresos Portão Ltda.

Lave, J. (2001). La práctica del aprendizaje. En Chaiklin y Lave (Comp.), Estudiar las prácticas. Perspectivas sobre actividad y contexto. Buenos Aires: Amorrortu editores.

Lion, C. (2006). Imaginar con tecnología. Relaciones entre tecnología y conocimiento. Buenos Aires: Editorial Stella.

Pintó, R., Couso, D. y Oro, J. (2006). A teaching approach about acoustics integrating different ICT and combining knowledge from different fields. En van den Berg, E, Ellermeijer, T. y Slooten, O. (Eds.), Modeling in Physics and Physics Education, Proceedings GIREP Conference 2006, 350-356.

Pontes Pedrajas, A. (2005). Aplicaciones de las tecnologías de la información y de la comunicación en la educación científica. Primera parte: funciones y recursos. Revista Eureka sobre Enseñanza y Divulgación de las Ciencias, Vol. 2, No 1, pp. 2-18. Recuperado el 02 de junio de 2009 de http://www.apaceureka.org/revista/

Pozo, J. I. y Gómez Crespo, M. A. (1998). Aprender y enseñar ciencia. Madrid: Ed. Morata/MEC.

Rodriguez Illera, J. L. (2004). El aprendizaje virtual: Enseñar y aprender en la era digital. Rosario: HomoSapiens. 
Teo, H., Oh, L., Liu, C. y Wei, K. (2003). An empirical study of the effects of interactivity on web user attitude. International Journal Human-Computer Studies, 58, 281-305. 


\section{APPLETS IN TEACHING PHYSICS: AN ANALYSIS OF THE TECHNOLOGIC AND DISCIPLINARY CHARACTERISTICS}

\section{SUMMARY}

An applet provides one scaffolding for conceptualizing dinamics phenomenons and it allows to connect the scientific model, mathematics and common sense. In this paper we describe both technological and disciplinary characteristics of waves applets, which have been selected on the Internet. It is has elaborated a set of categories to describe the interactive possibilities of the applets on the two aspects mentioned above. The results presented matches the first stage of analysis, and have showed a wide variety of elements that make interactions possible, ranging from the shows of graphics of variables to small microworlds. The elaborated categories can be a useful instrument for analyzing applets, and they could be considered as supplies for other educational research, as well as also they could be help to teachers in the process of select of simulations.

Keywords: Applet; interactivity; cognitive tools; scientific model; wave motion. 